\title{
A comparative study of anhedonia components between major depression and schizophrenia in Chinese populations
}

(1) CrossMark

Yinghui Li ${ }^{1}$, Xiaodong Mou' ${ }^{1}$, Wenhao Jiang ${ }^{1}$, Zhong Yang ${ }^{2}$, Xinhua Shen ${ }^{3}$, Zhuma Jin ${ }^{4}$, Zhiping Dai ${ }^{4}$, Yuju Liu ${ }^{5}$, Shengqin Mao ${ }^{1}$, Jian Zhang ${ }^{6}$ and Yonggui Yuan ${ }^{*}$

\begin{abstract}
Background: Anhedonia is a prominent symptom of major depressive disorder (MDD) and schizophrenia. At present, it is believed that hedonic processing rather consists of the anticipatory and consummatory phase. The aim of this research is to explore the different anhedonia components in MDD and schizophrenia in Chinese populations.

Methods: A Chinese version of the Temporal Experience of Pleasure Scale (TEPS) was used to evaluate 176 MDD patients, 346 schizophrenia patients, and 268 healthy controls. Additionally, the 17-item Hamilton Depression Rating Scale (HAMD-17) was used for MDD patients, while the Positive and Negative Syndrome Scale (PANSS) was applied for schizophrenia.

Results: The scores of consummatory (TEPS-CON) and anticipatory pleasure (TEPS-ANT) in MDD and schizophrenia were both significantly lower than healthy controls (both $\mathrm{P}<0.001$ ). TEPS-CON and TEPS-ANT were negatively correlated with the score of HAMD-17, the duration of illness and admission times in MDD ( $<<0.05$ or 0.01$)$. TEPS-CON was negatively related to PANSS total scores and negative symptoms ( $P<0.05$ or 0.01$)$, but no significant correlation was found with duration of illness and admission times in schizophrenia $(P>0.05)$. There was no significant correlation between TEPS-ANT and any clinical variables $(P>0.05)$.
\end{abstract}

Conclusions: The consummatory and anticipatory pleasures were both impaired in MDD and schizophrenia. Consummatory and anticipatory anhedonia can be considered as a "state" in MDD, but as a "trait" in schizophrenia.

Keywords: Anhedonia, Anticipatory pleasure, Consummatory pleasure, Major depressive disorder, Schizophrenia

\section{Background}

Anhedonia, the diminished capacity to experience pleasure, is a prominent symptom of major depressive disorder (MDD), schizophrenia and other neuropsychiatric disorders [1-4]. Almost 30 years ago, Klein [5] suggested that hedonic processing was not a unitary construct, but rather a complex of both anticipatory and consummatory components. Consummatory pleasure reflects the momentary pleasure that is experienced while engaged in

\footnotetext{
*Correspondence: yygylh2000@sina.com

1 Department of Psychosomatics and Psychiatry, ZhongDa Hospital, School of Medicine, Southeast University, Nanjing 210009, People's Republic of China

Full list of author information is available at the end of the article
}

an enjoyable activity, while anticipatory pleasure revolves around pleasure from future activities.

Anhedonia and depressed mood are the two core symptoms of MDD [1]. Hasler and colleagues [6] demonstrated that anhedonia together with increased stress reactivity was the most important candidate for psychopathological endophenotype of MDD. Joiner et al. [7] found that MDD patients presented higher scores on Beck Depression Inventory (BDI) anhedonic items than schizophrenic subjects, suggesting that anhedonia was a specific state-like feature of depressive illness. However, Pelizza and Ferrari [8] detected that anhedonia reached highly significant levels in $36.9 \%$ of depressed patients and $45 \%$ of schizophrenic patients according to the Physical Anhedonia Scale and the Social Anhedonia Scale, 
indicating that anhedonia could not be considered as a distinctive state in MDD.

Accumulating evidence suggested that depression was associated with anhedonia. Pizzagalli et al. [9] found blunted reward responsiveness in patients with MDD, particularly when anhedonic symptoms were prominent. Treadway et al. [10] observed an association between anhedonia and reduced motivation in an effort-based decision-making task in MDD. Liu et al. [11] utilizing the Snaith-Hamilton Pleasure Scale (SHAPS) in Chinese populations indicated that patients with depression demonstrated more anhedonic symptoms than patients with schizophrenia and non-clinical individuals. Although the above studies suggested diminished hedonic capacity in MDD, they did not differentiate consummatory and anticipatory anhedonia. To date, only one study distinguished consummatory and anticipatory anhedonia, stating low anticipatory pleasure but intact consummatory pleasure in depressed individuals [12].

In schizophrenia, anhedonia has long been considered a cardinal symptom [13] and a component of negative symptoms [14, 15]. Studies on anhedonia in schizophrenia have provided inconsistent results. Schizophrenia patients reported more severe anhedonia than healthy controls on self-report measures and semistructured interviews [16-18], but had normal levels of pleasant emotions while in response to pleasurable stimuli [19, 20]. Distinction of deficits in anticipatory and consummatory pleasure may help to understand the mixed findings on anhedonia in schizophrenia. A new self-report questionnaire, the Temporal Experience of Pleasure Scale (TEPS), developed by Gard et al. [21], was used to assess consummatory and anticipatory pleasure. Several studies showed anticipatory, but not consummatory anhedonia in schizophrenia [22-24]. However, the study of Strauss et al. [25] had the opposite result. Buck and Lysaker [26] suggested that anticipatory pleasure predicted concurrent and prospective levels of positive symptoms, emotional discomfort and interpersonal function, whereas consummatory pleasure predicted only concurrent positive symptoms.

In summary, little attention has been paid to detect the consummatory and anticipatory pleasure impairment in MDD, and there were inconsistent results on consummatory and anticipatory pleasure in schizophrenia. Moreover, there has been no study to compare the difference between MDD and schizophrenia on consummatory and anticipatory pleasure.

In this study, we assessed consummatory and anticipatory pleasure using TEPS in MDD and schizophrenia. The present study has two main aims: (1) examining the discrimination of consummatory and anticipatory pleasure between MDD or schizophrenia and healthy controls; and (2) exploring the relationships between different anhedonia types and clinical variables, including severity, duration of illness and admission times in MDD and schizophrenia.

\section{Methods \\ Participants}

This study included 176 patients with MDD, 346 patients with schizophrenia and 268 healthy controls. All the patients were inpatients who were recruited from six hospitals (one general hospital, five psychiatric hospitals): Zhongda Hospital affiliated to Southeast University, Changshu Mental Health Centre, Third People's Hospital of Hai'an, Brain Hospital affiliated to Nanjing Medical University, Fourth People's Hospital of Zhenjiang and Third People's Hospital of Huzhou. Clinical diagnoses were determined based on the Structured Clinical Interview for DSM-IV [1] by experienced psychiatrists. Patients with any other concurrent Axis I disorders were excluded; furthermore, patients with history of mental retardation, personality disorder, neurological disorders, such as stroke, head injury, seizure or diagnosis of alcohol or substance abuse/dependence in the last 6 months were also excluded from the study. All patients with MDD received antidepressant medication treatment, tricyclic antidepressants (TCAs, $\mathrm{n}=12$ ), selective serotonin reuptake inhibitors (SSRIs, $\mathrm{n}=86$ ), serotonin-norepinephrine reuptake inhibitors (SNRIs, $n=43$ ), noradrenergic and specific serotonergic antidepressant (NaSSA, $\mathrm{n}=25)$, other antidepressants $(\mathrm{n}=10)$ and two or more antidepressants $(\mathrm{n}=12)$. All patients with schizophrenia were treated with a regular dose of antipsychotic medications: typical $(\mathrm{n}=42)$, atypical $(\mathrm{n}=274)$ and mixed $(\mathrm{n}=30)$. Healthy controls were recruited from ZhongDa Hospital, including 216 health examination clients, 32 nurses and 20 doctors. All healthy controls were screened by experienced psychiatrists to ascertain that they did not have any neuropsychiatric symptoms.

\section{Measures \\ General Situation Questionnaire}

A self-designed questionnaire was used to investigate the general condition of participants, including demographical information, course of illness, admission times and type of medications.

\section{The Temporal Experience of Pleasure Scale (TEPS)}

The TEPS is composed of 18 items rated on a Likert-type scale ranging from 1 (Very True for me) to 6 (Very False for me) and yields two subscales measuring anticipatory (TEPS-ANT) and consummatory (TEPS-CON) pleasure. Lower scores indicate greater levels of anhedonia [21]. The Chinese version of TEPS has been proved to 
possess adequate validations and reliability in previous studies [24, 27]. The current study found that TEPSANT and TEPS-CON were positively correlated with the General Self-efficacy Scale [28] $(r=0.27,0.25$, separately, $\mathrm{P}<0.01)$ and inversely related with the BDI [29] (TEPS-ANT $r=-0.19,-0.13$, separately, $\mathrm{P}<0.01$ ). The correlation between the TEPS and other scales suggested that the TEPS had adequate convergent and discriminant validity. In this study, the Chinese version of TEPS had demonstrated good internal consistency (TEPS-ANT, alpha $=0.706$; TEPS-CON, alpha $=0.702)$ and testretest reliability (TEPS-ANT, $r=0.698$; TEPS-ANT, $\mathrm{r}=0.615$ ).

\section{7-item Hamilton Depression Rating Scale (HAMD-17)}

Depression severity was measured with the Chinese version HAMD-17 in depression patients. A baseline HAMD-17 score $\geq 17$ was applied as an inclusion criterion. The Chinese version of HAMD-17 has demonstrated good internal consistency and test-retest reliability [30].

\section{Positive and Negative Syndrome Scale (PANSS)}

PANSS was applied to evaluate the clinical severity of positive, negative and other symptoms in schizophrenia. It is a 30 -item scale, in which 7 constitute a positive scale, 7 a negative scale and the remaining 16 a general psychopathology scale. Each item contains seven rating points representing increasing levels of psychopathology. The Chinese version of PANSS has demonstrated good internal consistency (alpha 0.73-0.83 depending on the subscale) [31].

\section{Procedure}

All participants for this study filled out the TEPS. Additionally, patients with major depression completed HAMD-17 to measure depression severity, and patients with schizophrenia completed PANSS to measure clinical symptoms. All participations were given written informed consent to participate in the study. The study procedure was approved by the Ethical Committee of ZhongDa Hospital affiliated to Southeast University, China.

\section{Statistical analyses}

Categorical variables were compared by means of the Chi-square test. One-way analysis of variance (ANOVA) was employed to compare continuous variables among groups, and post hoc LSD tests were performed in cases of significant ANOVA effects. Pearson correlations were conducted to assess relationships between anhedonia and clinical variables. Statistical analyses were performed using the SPSS program version 11.5.

\section{Results}

Participants' demographic and clinical characteristics

The participants' demographic and clinical characteristics are reported in Table 1. There was no significant difference among these three groups in $\operatorname{gender}\left(\chi^{2}=2.01\right.$, $P>0.01)$, age $(\mathrm{F}=1.73, P>0.01)$ and the level of education $(\mathrm{F}=1.75, P>0.01)$.

\section{Comparisons between patients with MDD or schizophrenia and healthy controls}

One-way ANOVA indicated significant differences in the TEPS-ANT and TEPS-CON scores $(\mathrm{F}=40.8,89.1$ respectively, $P<0.001$ ) among the three groups. Post hoc tests showed that TEPS-ANT and TEPS-CON scores in MDD and schizophrenia were both lower than those of healthy controls (all $P<0.01$ ), and TEPS-ANT and TEPS-CON scores in patients with MDD were lower $(P<0.01$ or 0.05 , respectively) than in those with schizophrenia (Fig. 1).

Table 1 Socio-demographic and clinical characteristics of the study population

\begin{tabular}{llll}
\hline & $\begin{array}{l}\text { Patients with } \\
\text { MDD }(\boldsymbol{n = 1 7 6 )}\end{array}$ & $\begin{array}{l}\text { Patients with schizophrenia } \\
(\boldsymbol{n = 3 4 6 )}\end{array}$ & $\begin{array}{l}\text { Heathy controls } \\
(\boldsymbol{n}=\mathbf{2 6 8})\end{array}$ \\
\hline Age (year) & $35.51 \pm 11.88$ & $33.75 \pm 10.22$ & $34.10 \pm 9.47$ \\
Females/males & $98 / 79$ & $178 / 168$ & $135 / 133$ \\
Education (year) & $10.86 \pm 3.01$ & $10.62 \pm 2.48$ & $11.08 \pm 3.64$ \\
Duration of illness (year) & $5.71 \pm 8.98$ & $8.10 \pm 8.79$ & - \\
Admission times & $2.20 \pm 1.82$ & $3.53 \pm 3.31$ & - \\
HAMD-17 & $16.12 \pm 8.05$ & - & - \\
PANSS total & - & $69.09 \pm 22.04$ & - \\
PANSS positive & - & $16.70 \pm 7.40$ & - \\
PANSS negative & - & $18.04 \pm 6.98$ & - \\
PANSS general & - & $33.69 \pm 11.11$ & - \\
\hline
\end{tabular}




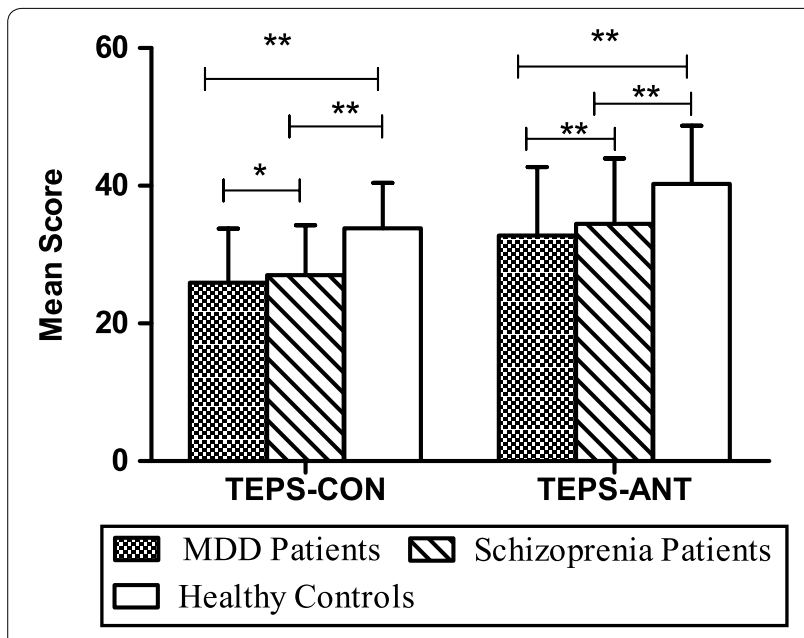

Fig. 1 Comparisons of TEPS-CON and TEPS-ANT between patients with MDD or schizophrenia and healthy controls. ${ }^{*} P<0.05$, ${ }^{* *} P<0.01$. One-way ANOVA indicated significant differences in the TEPS-ANT and TEPS-CON scores (both $P<0.001$ ) among the three groups. Post hoc tests showed that TEPS-ANT and TEPS-CON scores in MDD and schizophrenia were both lower than those in healthy controls (all $P<0.01$ ), and TEPS-ANT and TEPS-CON scores in MDD were lower $(P<0.01$ or 0.05 respectively) than those in schizophrenia

\section{Associations between TEPS subscales and clinical variables in patients with MDD}

Table 2 presents that both TEPS-CON and TEPS-ANT scores were negatively correlated with HAMD-17 scores, duration of illness and admission times in patients with MDD $(P<0.05$ or 0.01$)$.

\section{Associations between TEPS subscales and clinical variables in patients with schizophrenia}

As shown in Table 3, TEPS-CON scores were inversely correlated with PANSS total scores, and positive and negative symptoms $(P<0.05$ or 0.01$)$ in schizophrenia. There was no significant correlation between TEPS$\mathrm{CON}$ and general psychopathology, duration of illness or admission times $(P>0.05)$. TEPS-ANT showed no significant correlation with all clinical characteristics (PANSS total scores, positive symptoms, negative symptoms, general psychopathology, duration of illness and admission times) in schizophrenia patients.

Table 2 The Pearson correlation coefficients between TEPS and clinical characteristics in patients with MDD

\begin{tabular}{lll}
\hline & TEPS-CON & TEPS-ANT \\
\hline HAMD-17 & $-0.48^{* *}$ & $-0.54^{* *}$ \\
Duration of illness (years) & $-0.18^{*}$ & $-0.21^{* *}$ \\
Admission times & $-0.21^{* *}$ & $-0.17^{*}$
\end{tabular}

${ }^{*} P<0.05$

** $P<0.01$
Table 3 The Pearson correlation coefficients between TEPS subscales and clinical characteristics in patients with schizophrenia

\begin{tabular}{llc}
\hline & TEPS-CON & TEPS-ANT \\
\hline PANSS total & $-0.14^{* *}$ & -0.07 \\
PANSS positive & $-0.11^{*}$ & -0.09 \\
PANSS negative & $-0.78^{* *}$ & 0.06 \\
PANSS general & -0.10 & 0.07 \\
Duration of illness (years) & -0.004 & -0.08 \\
Admission times & 0.03 & -0.08 \\
\hline
\end{tabular}

* $P<0.05$

** $P<0.01$

\section{Discussion}

The present study indicated that both consummatory and anticipatory pleasure were impaired in MDD. The results were not completely consistent with most previous studies which usually reflected either consummatory anhedonia $[7,8,11]$ or anticipatory pleasure impaired by depression [12]. It is noteworthy that several theorists have proposed that anhedonic features are related to altered dopaminergic circuitry in MDD [32-34]. Particularly, the dysfunction of dopaminergic mesolimbic and mesocortical reward circuits has been singled out as the major neurobiological correlate of anhedonia [35, 36]. Mesolimbic dopaminergic neurons projecting into the frontal cortex and nucleus accumbens have been suggested to be involved in the therapeutic actions of some antidepressants [37, 38], through 5-HT modulation of DA function [39]. Previous studies have shown that antidepressants can influence anhedonia in patients with MDD in dopaminergic mesolimbic reward circuits likely caused by the increase in serotonin neurotransmission $[38,40,41]$. We speculate that the difference between the present study and other studies may be due to the influence of antidepressants on anhedonia.

This study also found anticipatory and consummatory pleasure impaired in schizophrenia. Gard et al. [22] and Chan et al. [24] observed lower anticipatory pleasure in schizophrenia compared with healthy controls. But Strauss et al. [25] found lower score on consummatory pleasure. Our findings were not completely consistent with the mentioned studies. Strauss et al. [25] supposed that the main difference between their study and the study of Gard et al. and Chan et al. was in the percentage of patients who were prescribed typical antipsychotics. We agreed with this speculation. In the present study, $21 \%$ of patients were prescribed typical antipsychotics, compared to $31 \%$ in Gard et al. $29 \%$ in Chan et al. and $12 \%$ in Strauss et al. Previous evidence showed that typical antipsychotics could produce anhedonia via 
dopamine antagonism [42], and a neurobiological study showed that dopamine was strongly linked to anticipatory rather than consummatory pleasure $[43,44]$. In contrast, consummatory pleasure was more strongly linked to the serotonin and opioid systems $[45,46]$. Further evidence also revealed that typical antipsychotics lead to more reward prediction dysfunction [47], and therefore anticipatory rather than consummatory pleasure was even more easily influenced by typical antipsychotics. Another reason for this might be that previous studies missed some factors such as relapse times, acute or chronic phase and patients in hospitals or community homes $[22,24,25]$.

In the correlation study, we found diminished consummatory and anticipatory pleasure associated with depression severity in patients with MDD. The results were in concordance with other studies showing that the severity of anhedonia was positively correlated with the severity of depressive symptoms in MDD [48-52]. We also found that TEPS-CON and TEPS-ANT had significant positive correlations with the duration of illness and admission times in MDD. The results indicated that impaired consummatory and anticipatory pleasure resulted in worse prognosis of MDD. It was consistent with the opinion that anhedonia was a post-depressive "scar" symptom in MDD [53].

In previous studies, anhedonia did not show strong association with the duration of illness, number of admissions, cumulative time in hospital and length of current admission in schizophrenia. It remains fairly stable over time and is generally considered to reflect as a "trait" rather than a "state" in schizophrenia $[15,54,55]$. However, whether anticipatory and consummatory anhedonia would be stable over time in schizophrenia was unclear. The current results indicated that TEPS-ANT and TEPSCON scores had no significant relationship with the course of illness and admission times, which suggested that both anticipatory and consummatory anhedonia remained relatively stable in schizophrenia.

Herbener and Harrow found significant correlations between anhedonia and negative symptoms, but no meaningful relationships with positive symptoms in schizophrenia in a 10-year longitudinal study [56]. However, other studies indicated significant associations between anhedonia and positive symptoms $[57,58]$. No statistically significant associations between anhedonia and positive, negative and general psychopathology scores as measured by PANSS were detected in the research of Huxley et al. [59]. In this study, we found that TEPS-CON significantly correlated with PANSS total scores, negative and positive symptoms, but TEPS-ANT had no meaningful relationship with any symptoms. The results suggested that consummatory anhedonia might vary with the severity of negative and positive symptoms, but anticipatory anhedonia seemed to be independent of many clinical variables, such as positive and negative symptoms. This seemingly showed that anticipatory anhedonia was more stable than consummatory pleasure deficit in patients with schizophrenia.

There were some limitations in this study. First, the sample of patients was recruited from clinical randomly as long as the patients met the inclusion criteria. All the patients in our study were taking medication; although the differences among medications were taken into account, we did not consider the influence of different doses on anhedonia in patients with MDD and schizophrenia. Second, different sites and inter-rater bias could influence the measurement results. Finally, this study did not measure TEPS in different clinical phases to understand whether consummatory and anticipatory pleasure would vary with time.

\section{Conclusions}

Consummatory and anticipatory anhedonia were both found in MDD and schizophrenia. The two anhedonia components were significantly associated with depression severity, duration of illness and admission times in MDD. Therefore, we speculated that both consummatory and anticipatory pleasure deficit could be regarded as a "state" in MDD. However in schizophrenia, consummatory anhedonia was only associated with negative and positive symptoms, and anticipatory anhedonia had no significant correlation with any clinical characteristics. So, anticipatory and consummatory anhedonia can be considered as a "trait", and it seemed more stable than consummatory anhedonia in schizophrenia.

\section{Abbreviations}

DSM-IV: Diagnostic and Statistical Manual of Mental Disorders Criteria-fourth edition; SHAPS: Snaith-Hamilton Pleasure Scale; BDI: Beck Depression Inventory; TEPS: Temporal Experience of Pleasure Scale; TEPS-CON: consummatory pleasure in Temporal Experience of Pleasure Scale; TEPS-ANT: anticipatory pleasure in Temporal Experience of Pleasure Scale; HAMD-17: 17-item Hamilton Depression Rating Scale; PANSS: Positive and Negative Syndrome Scale.

\section{Authors' contributions}

YY conceived the study and the design, translated TEPS into Chinese and helped to draft and revise the manuscript. YLi participated in its design, conducted the statistical analyses and wrote the manuscript. WJ participated in its design, the data acquisition and the scale translation. ZY, XS, ZJ, ZD, YLiu, $\mathrm{SM}$ and JZ coordinated the study and data acquisition. All authors read and approved the final manuscript.

\section{Author details}

${ }^{1}$ Department of Psychosomatics and Psychiatry, ZhongDa Hospital, School of Medicine, Southeast University, Nanjing 210009, People's Republic of China.

2 Department of Psychiatry, Changshu Mental Health Centre, Changshu, China

${ }^{3}$ Department of Psychosomatics, The Third People's Hospital of Huzhou, Huzhou, China. ${ }^{4}$ Department of Psychiatry, Brain Hospital affiliated to Nanjing Medical University, Nanjing, China. ${ }^{5}$ Department of Psychiatry, The Fourth People's Hospital of Zhenjiang, Zhenjiang, China. ${ }^{6}$ Department of Psychiatry, The Third People's Hospital of Haian, Haian, China. 


\section{Acknowledgements}

This study was supported by a grant from the National Natural Science Foundation of China (Grant No: 81371488 Yonggui Yuan).

\section{Compliance with ethical guidelines}

\section{Competing interests}

The authors declare that they have no competing interests.

Received: 19 January 2015 Accepted: 20 August 2015

Published online: 03 September 2015

\section{References}

1. American Psychiatric Association (1994) Diagnostic and statistical manual of mental disorders, 4th edn. American Psychiatric Association, Washington, DC

2. Juckel G, Sass L, Heinz A (2003) Anhedonia, self-experience in schizophrenia, and implications for treatment. Pharmacopsychiatry 36:176-180

3. Loas G, Noisette C, Legrand A, Boyer P (2000) Is anhedonia a specific dimension in chronic schizophrenia? Schizophr Bull 26:495-506

4. Nagayamal H, Kubo S, Hatano T, Shinsuke H, Tetsuya M, Takafumi H, Taro K, Hiroo T, Masayuki Y, Nobuatsu N, Osamu K, Manabu I, Hideki S, Tatsuya T, Tsuyoshi U, Hirohisa W, Satoshi K (2012) Validity and reliability assessment of a Japanese version of the Snaith-Hamilton Pleasure Scale. Intern Med 51:865-869

5. Klein D (1984) Depression and anhedonia. In: Clark DC, Fawcett J (eds) Anhedonia and affect deficit states. PMA Publishing, New York, pp $1-14$

6. Hasler G, Drevets WC, Manji HK, Charney DS (2004) Discovering endophenotypes for major depression. Neuropsychopharmacol 29:1765-1781

7. Joiner T, Brown J, Metalsky G (2003) A test of the tripartite model's prediction of anhedonia's specificity to depression: patients with major depression versus patients with schizophrenia. Psychiatry Res 119:243-250

8. Pelizza L, Ferrari A (2009) Anhedonia in schizophrenia and major depression: state or trait? Ann Gen Psychiatry 8:22

9. Pizzagalli DA, losifescu D, Hallett LA, Ratner KG, Fava M (2008) Reduced hedonic capacity in major depressive disorder: evidence from a probabilistic reward task. J Psychiatr Res 43:76-87

10. Treadway MT, Buckholtz JW, Schwartzman AN, Lambert WE, Zald DH (2009) Worth the 'EEfRT'? The effort expenditure for rewards task as an objective measure of motivation and anhedonia. PLoS One 4:e6598

11. Liu WH, Wang LZ, Zhu YH, Li MH, Chan RCK (2012) Clinical utility of the Snaith-Hamilton-Pleasure scale in the Chinese settings. BMC Psychiatry 12:184

12. Sherdell L, Waugh CE, Gotlib IH (2012) Anticipatory pleasure predicts motivation for reward in major depression. J Abnorm Psychol 121:51-60

13. Blanchard JJ, Mueser KT, Bellack AS (1998) Anhedonia, positive and negative affect, and social functioning in schizophrenia. Schizophr Bull 24:413-424

14. Loas G, Boyer P, Legrand A (1999) Anhedonia and the deficit syndrome of schizophrenia. Psychopathology 32:207-219

15. Horan WP, Kring AM, Blanchard JJ (2006) Anhedonia in schizophrenia: a review of assessment strategies. Schizophr Bull 32:259-273

16. Silver H, Shlomo N (2012) Anhedonia and schizophrenia: how much is in the eye of the beholder? Compreh Psychiatry 43:65-68

17. Berenbaum H, Fujita F (1994) Schizophrenia and personality: Exploring the boundaries and connections between vulnerability and outcome. J Abnorm Psychol 103:148-158

18. Horan WP, Blanchard JJ, Clark LA, Green MF (2008) Affective traits in schizophrenia and schizotypy. Schizophr Bull 34:856-874

19. Cohen AS, Minor KS (2010) Emotional experience in patients with schizophrenia revisited: meta-analysis of laboratory studies. Schizophr Bull 36:143-150

20. Kring AM, Moran EK (2008) Emotional response deficits in schizophrenia: Insights from affective science. Schizophr Bull 34:819-834

21. Gard DE, Gard MG, Kring AM, John OP (2006) Anticipatory and consummatory components of the experience of pleasure: a scale development study. J Res In Personal 40:1086-1102
22. Gard DE, Kring AM, Gard MG, Horan WP, Green MF (2007) Anhedonia in schizophrenia:distinctions between anticipatory and consummatory pleasure. Schizophr Res 93:253-260

23. Favrod J, Ernst F, Giuliani F, Bonsack C (2009) Validation of the temporal experience of pleasure scale (TEPS) in a French-speaking environment. Encephale 35:241-248

24. Chan RCK, Wang Y, Huang J, Shi Y, Wang Y, Hong X, Ma Z, Li Z, Lai MK, Kring AM (2010) Anticipatory and consummatory components of the experience of pleasure in schizophrenia: cross-cultural validation and extension. Psychiatry Res 175:181-183

25. Strauss GP, Wilbur RC, Warren KR, August SM, Gold JM (2011) Anticipatory vs. consummatory pleasure: What is the nature of hedonic deficitsin schizophrenia? Psychiatry Res 187:36-41

26. Buck B, Lysaker PH (2013) Consummatory and anticipatory anhedonia in schizophrenia: stability, and associations with emotional distress and social function over six months. Psychiatry Res 205:30-35

27. Chan RCK, Shi Y, Lai M, Wang YN, Wang Y (2012) The temporal experience of pleasure scale (TEPS): exploration and confirmation of factor structure in a healthy chinese sample. PLoS ONE 7:e35352

28. Beck AT, Ward CH, Mendelson M, Mock J, Erbaugh J (1961) An inventory for measuring depression. Arch Gen Psychiatry 4:561-571

29. Jerusalem M, Schwarzer R (1992) Self-efficacy as a resource factor in stress appraisal processes. Hemisphere, Washington DC

30. Tang YH (2005) Hamilton Depression Scale. In: Zhang ZJ (ed) Behavioral medicine inventory manual. The Chinese Medicine Electronic Audio And Video Publishing House, Beijing, pp 224-227

31. He YL (2005) Positive and Negative Syndrome Scale. In: Zhang ZJ (ed) Behavioral medicine inventory manual. The Chinese Medicine Electronic Audio And Video Publishing House, Beijing, pp 355-359

32. Pizzagalli DA, Jahn AL, O'shea JP (2005) Toward an objective characterization of an anhedonia phenotype: a signal-detection approach. Biol Psychiatry 57:319-327

33. Gershon AA, Vishne T, Grunhaus L (2007) Dopamine D2-like recepters and the antidepressant response. Biol Psych: Blunted response to feedback information in depressive illness. Brain 130:2367-2374

34. Steele JD, Kumar P, Ebmeier KP (2007) Blunted response to feedback information in depressive illness. Brain 130:2367-2374

35. Willner P, Hale AS, Argyropoulos S (2005) Dopaminergic mechanism of antidepressant action in depressed patients. J Affect Disord 86:37-45

36. Gorwood P (2008) Neurobiological mechanisms of anhedonia. Dialogues Clin Neurosci 10:291-299

37. Kapur S, Mann JJ (1992) Role of the dopaminergic system in depression. Biol Psychiatry 32:1-17

38. Nestler EJ, Carlezon WA (2006) The mesolimbic dopamine reward circuit in depression. Biol Psychiatry 59:1151-1159

39. Barnes NM, Sharp T (1999) A review of central 5-HT receptors and their function. Neuropharmacology 38:1083-1152

40. Yan QS (2000) Activation of 5-HT2A/2C receptors within the nucleus accumbens increases local dopaminergic transmission. Brain Res Bull 51:75-81

41. Marutani T, Yahata N, Ikeda Y, Ito T, Yamamoto M, Matsuura M, Matsushima E, Okubo Y, Suzuki H, Matsuda T (2011) Functional magnetic resonance imaging study on the effects of acute single administration of paroxetine on motivation-related brain activity. Psychiatry Clin Neurosci 65:191-198

42. Awad AG, Voruganti LN (2005) Neuroleptic dysphoria: revisiting the concept 50 years later. Acta Psychiatr Scand 111(Suppl):6-13

43. Berridge KC, Robinson TE (1998) What is the role of dopamine in reward: Hedonic impact, reward learning, or incentive salience? Brain Res Rev 28:309-369

44. Berrridge KC (2007) The debate over dopamine's role in reward: The case for incentive salience. Psychopharmacol 191:391-431

45. SchultzW (2002) Getting formal with dopamine and reward. Neuron $36: 241-263$

46. Wise RA (2002) Brain reward circuitry: Insights from unsensed incentives. Neuron 36:229-240

47. Juckel G, Schlagenhauf F, Koslowski M, Wustenberg T, Villringer A, Knutson B, Kienast T, Gallinat J, Wrase J, Heinz A (2006) Dysfunction of ventral striatal reward prediction in schizophrenia. Neuroimage 29:409-416 
48. Mathews JR, Barch DM (2006) Episodic memory for emotional and non-emotional words in individuals with anhedonia. Psychiatry Res 143:121-133

49. Chentsova-Dutton Y, Hanley K (2010) The effects of anhedonia and depression on hedonic responses. Psychiatry Res 179:176-180

50. Kaviani H, Gray J, Checkley S, Raven P, Wilson G, Kumari V (2004) Affective modulation of the startle response in depression: influence of the severity of depression, anhedonia, and anxiety. J Affect Disord 83:21-31

51. Leventhal AM, Chasson GS, Tapia E, Miller E, Pettit JW (2006) Measuring hedonic capacity in depression: a psychometric analysis of three anhedonia scales. J Clin Psychol 62:1545-1558

52. Vollmayr B, Henn FA (2003) Stress models of depression. Clin Neurosci Res 3:245-251

53. Fawcett J, Clark D, Scheftner A (1982) Differences between anhedoniaand normal hedonic depressive states. Am J Psychiatry 140:1027-1030

54. Silver H, Shlomo N (2002) Anhedonia and schizophrenia: how much is in the eye of the beholder? Compreh Psychiatry 43:65-68
55. Rey ER, Bailer J, Brauer W, Händel M, Laubenstein D, Stein A (1994) Stability trends and longitudinal correlations of negative and positive syndromes within a three-year follow-up of initially hospitalized schizophrenics. Acta Psychiat Scand 90:405-412

56. Herbener ES, Harrow M (2002) The course of anhedonia during 10 years of schizophrenic illness. J Abnorm Psychol 111:237-248

57. Kontaxakis VP, Kollias CT, Havaki-Kontaxaki BJ, Margariti MM, Stamouli SS, Petridou E, Christodoulou GN (2006) Physical anhedonia in the acute phase of schizophrenia. Ann Gen Psychiatry 5:1

58. Brebion G, David AS, Jones H, Pilowsky LS (2005) Hallucinations, negative symptoms, and response bias in a verbal recognition task in schizophrenia. Neuropsychol 19:612-617

59. Huxley A, Fonseca AS (2014) The relationship between anhedonia and positive, negative, and general symptomatology in patients with schizophrenia. Ment Health Nurs 35:122-126

\section{Submit your next manuscript to BioMed Central and take full advantage of:}

- Convenient online submission

- Thorough peer review

- No space constraints or color figure charges

- Immediate publication on acceptance

- Inclusion in PubMed, CAS, Scopus and Google Scholar

- Research which is freely available for redistribution

Submit your manuscript at

www.biomedcentral.com/submit

C Biomed Central 